\title{
Anti-MAdCAM-1 antibody (PF-00547659) for active refractory Crohn's disease in Japanese and Korean patients: the OPERA study
}

\author{
Masayuki Saruta ${ }^{1}$, Dong Il Park ${ }^{2}$, Young-Ho Kim³ ${ }^{3}$, Suk-Kyun Yang ${ }^{4}$, Byung-Ik Jang ${ }^{5}$, Jae Hee Cheon ${ }^{6}$, Jong Pil Im \\ Takanori Kanai $^{8}$, Tatsuro Katsuno ${ }^{9}$, Yoh Ishiguro ${ }^{10}$, Makoto Nagaoka ${ }^{11}$, Naoki Isogawa ${ }^{12}$, Yinhua Li $^{13}$, \\ Anindita Banerjee ${ }^{14}$, Alaa Ahmad ${ }^{15}$, Mina Hassan-Zahraee ${ }^{16}$, Robert Clare ${ }^{17}$, Kenneth J. Gorelick ${ }^{17}$, Fabio Cataldi ${ }^{18}$, \\ Mamoru Watanabe ${ }^{19}$, Toshifumi Hibi ${ }^{20}$ \\ ${ }^{I}$ Division of Gastroenterology and Hepatology, Department of Internal Medicine, The Jikei University School of Medicine, Tokyo, Japan; \\ ${ }^{2}$ Department of Internal Medicine, Kangbuk Samsung Hospital, Sungkyunkwan University School of Medicine, Seoul; ${ }^{3}$ Department of \\ Medicine, Samsung Medical Center, Sungkyunkwan University School of Medicine, Seoul; ${ }^{4}$ Department of Gastroenterology, Asan Medical \\ Center, University of Ulsan College of Medicine, Seoul; ${ }^{5}$ Department of Internal Medicine, Yeungnam University College of Medicine, Daegu; \\ ${ }^{6}$ Institute of Gastroenterology and Department of Internal Medicine, Yonsei University College of Medicine, Seoul; ${ }^{7}$ Division of Gastroenterology, \\ Department of Internal Medicine, Seoul National University Hospital, Seoul, Korea; ${ }^{8}$ Department of Internal Medicine, Keio University \\ Hospital, Tokyo; ${ }^{9}$ Department of Gastroenterology, Chiba University Hospital, Chiba; ${ }^{10}$ Department of Gastroenterology and Hematology, \\ National Hospital Organization, Hirosaki National Hospital, Hirosaki, ${ }^{11}$ Clinical Research, ${ }^{12}$ Clinical Statistics, and ${ }^{13}$ Clinical Pharmacology, \\ Development Japan, Pfizer Japan Inc., Tokyo, Japan; ${ }^{14}$ Clinical Statistics, ${ }^{15}$ Gastroenterology, and ${ }^{16}$ Early Clinical Research and Development, \\ Pfizer Inc., Cambridge, MA; ${ }^{17}$ Biotherapeutics Clinical R\&D, Pfizer Inc., Collegeville, PA; ${ }^{18}$ Gastroenterology, Clinical Programs, Pfizer Inc., \\ Cambridge, MA, USA; ${ }^{19}$ Department of Gastroenterology and Hepatology, Tokyo Medical and Dental University, Tokyo; ${ }^{20}$ Center for Advanced \\ IBD Research and Treatment, Kitasato Institute Hospital, Kitasato University, Tokyo, Japan
}

Background/Aims: PF-00547659 is a monoclonal antibody against human mucosal addressin cell adhesion molecule-1 (MAdCAM-1) that prevents the binding of $\alpha 4 \beta 7^{+}$lymphocytes to MAdCAM-expressing sites in the gastrointestinal tract with high affinity and selectivity, and is being developed for the treatment of Crohn's disease (CD). Methods: OPERA is a randomized, multicenter, double-blind, placebo-controlled study to investigate the efficacy, safety, and pharmacokinetics of PF-00547659 following subcutaneous administration in subjects with active CD, a history of failure or intolerance to anti-tumor necrosis factor and/or immunosuppressants, high-sensitivity C-reactive protein $>3.0 \mathrm{mg} / \mathrm{L}$, and ulcers on colonoscopy. The primary endpoint was Crohn's Disease Activity Index-70 response at week 8 or 12. Subpopulation analyses for Asian subjects were performed as some differences are observed in genetics and clinical phenotypes in Asian CD patients compared with Western patients. Results: In this study, 265 CD subjects were randomized, with a subpopulation of 21 subjects (8 Japanese and 13 Korean) defined as the Asian population. In the overall and Asian populations; PF-00547659 was pharmacologically active as evidenced by soluble MAdCAM and circulating $\beta 7^{+}$central memory CD4+ T-lymphocytes, although no clear evidence of efficacy was observed in any clinical endpoints; pharmacokinetics of PF-00547659 in the Asian subpopulation was generally comparable to the overall population; and the safety profile of PF-00547659 appeared acceptable up to 12 weeks of treatment. Conclusions: In the overall and Asian populations, efficacy of PF-00547659 could not be demonstrated using any clinical endpoints compared with placebo. Pharmacokinetics and safety of PF-00547659 were generally comparable. Further studies with larger numbers of patients are required to confirm our results. (Trial Registration Number: NCT01276509) (Intest Res 2020;18:45-55)

Key Words: PF-00547659; MAdCAM; Crohn disease; Japanese; Korean 


\section{INTRODUCTION}

The pathogenesis of $\mathrm{CD}$ is characterized by dysregulation of the immune response to antigen stimulation in the GI tract, with differences between individuals in genetic susceptibility. The major treatments for CD are immunotherapies such as corticosteroids, immunosuppressants, and anti-TNF inhibitors. ${ }^{1}$ Since there are many secondary nonresponders to these treatments, novel drug therapies are required. In the search for novel treatments, inhibition of cell adhesion molecules has received attention as a novel approach in the treatment of IBD.

Vedolizumab, a monoclonal antibody directed against the $\alpha 4 \beta 7$ integrin, has been approved in many countries for the treatment of patients with moderate to severe CD or UC whose response to existing therapies has been inadequate, has diminished, or has indicated that they were intolerant.

Mucosal addressin cell adhesion molecule (MAdCAM), expressed on gut endothelium, plays a role in gut immune surveillance and also appears to facilitate excessive lymphocyte infiltration under conditions of chronic GI inflammation. ${ }^{2-4}$ PF-00547659 is an anti-MAdCAM monoclonal antibody that prevents the binding of $\alpha 4 \beta 7^{+}$lymphocytes to MAdCAM-expressing sites in the high endothelial venules of lymphoid tissue of the GI tract with high affinity and selectivity. ${ }^{5}$ It is being developed for the treatment of CD and UC. OPERA was a randomized, multicenter, double-blind, placebo-controlled study to investigate the efficacy, safety, pharmacokinetics (PK), and pharmacodynamics of PF-00547659 following subcutaneous administration in 265 CD subjects, including 21 Asian subjects enrolled in Korea and Japan (13 Korean and 8 Japanese). ${ }^{6}$

There are some differences between Asian and Western populations with respect to the epidemiology, clinical characteristics, and use of therapies for $\mathrm{CD} .{ }^{7}$ For example, the clinical presentation of CD in Asia is predominantly ileo-colonic, with lower rates of familial aggregation compared with the West. ${ }^{7}$ In terms of therapies, granulocyte and monocyte adsorptive apheresis (GMA) is an approved therapy in Japan for CD patients refractory to anti-TNFs or immunosuppressants, whereas GMA has not demonstrated effectiveness in inducing clinical remission or response in Western patients with moderate to severe $\mathrm{CD}{ }^{8}{ }^{8}$ In general, there are race-associated differences in clinical characteristics and responses to existing therapeutic options for many diseases. Thus, race-based subpopulation analyses could provide a better understanding of efficacy and safety of a novel drug for treatment of the disease. Genetic studies have also shown that the phenotypes and clinical course of IBD are likely to differ between Asian and European patients. ${ }^{9}$ With this in mind, we performed subpopulation analyses for Japanese and Korean subjects in the OPERA study to characterize efficacy, safety, and PK profiles of PF00547659 and to compare these results with those of the overall study population.

\section{METHODS}

\section{Study Design}

This was a phase 2, randomized, double-blind, placebo-controlled, parallel-group trial in adult (ages 18-75 years) subjects with moderate to severe refractory $\mathrm{CD}$ who failed, or were intolerant to, treatment with immunosuppressants or anti-TNF agents. ${ }^{6}$ Eligible patients were randomly assigned in a 1:1:1:1 ratio to one of 4 treatment groups: each received blinded subcutaneous injections of placebo, $22.5 \mathrm{mg}, 75 \mathrm{mg}$, or $225 \mathrm{mg}$ of PF-00547659 (Pfizer, New York, NY, USA) at weeks 0, 4, and 8, and were followed through week 12 . Randomization was performed centrally according to a computer-generated scheme and stratified to 1 of 16 categories by the status of treatment failure with, or intolerance to, anti-TNF agents/immunosuppressant therapies (4 categories: anti-TNF relapse, anti-TNF primary nonresponder, anti-TNF intolerant, or failure/intolerance to any immunosuppressant) and concomitant immunosuppressive therapy type (4 categories: azathioprine, 6-mercaptopurine, methotrexate, or none). Stable doses of immunosuppressants were continued from screening through week 8. Beginning at week 8 , prior immunosuppressant dosage could be tapered by $\sim 25 \%$ per week to ensure that immunosuppressive therapy was discontinued by week 12 . Patients had the opportunity to participate in an open-label extension study if they satisfied the relevant eligibility criteria. The study was conducted in accordance with the Declaration of Helsinki and with the approval of the relevant ethics committee at each site. All patients provided written informed consent.

\section{Efficacy Evaluations}

The primary efficacy outcome was CDAI-70 clinical response, defined as a decrease from baseline in the CDAI score $\geq 70$ points at week 8 or week 12 . Secondary efficacy outcomes, measured from weeks 2 to 12, were as follows: the proportions of patients in CDAI-70 response; the proportions of patients in CDAI-100 clinical response (100-point decrease from the week 0 CDAI score); the proportions in CDAI remission (CDAI score < 150); and the total CDAI score and the mean change 
from week 0 in the total CDAI score by visit. Other secondary outcomes were the fecal calprotectin and high-sensitivity CRP (hs-CRP) concentrations at weeks $0,4,8$, and 12 , and the change from week 0 in fecal calprotectin and hs-CRP concentrations at weeks 4, 8, and 12. Post hoc analyses were conducted for the CDAI-70 and - 100 responses and the CDAI remission endpoints for subgroups of patients with baseline hs-CRP concentrations of $>5.0$ and $>18.8 \mathrm{mg} / \mathrm{L}$ (median), and with baseline Simple Endoscopic Activity Score-CD (SES-CD) of $>10$ (25th percentile) and $>17$ (median). It should be noted that for some patients, the endoscopy was performed up to 8 weeks prior to randomization. For these patients, the SES-CD score was calculated using data from that endoscopy, while for the remaining patients the SES-CD was determined at the time of baseline colonoscopy.

\section{Safety Evaluations}

Adverse events (AEs), concomitant medications, and clinical laboratory parameters were recorded throughout the study. Serum samples for antidrug antibodies (ADAs) were collected every 4 weeks during the induction period. Analysis of ADAs was conducted on serum samples using a multi-tiered approach including the screening assay, confirmatory assay, and neutralizing antibody assay.

\section{PK Evaluations}

PK samples were collected every 2 weeks during the induction period (up to 12 weeks). In order to characterize the full PK profile, additional PK samples were collected on days 3, 7 , 10 , and 21 for a subset of subjects excluding Japanese subjects, and on days 1 (1,6, and 24 hours post dose), 7 , and 21 after the first dose for Japanese subjects. PF-00547659 serum PK concentrations were analyzed using a validated, sensitive, and specific ELISA method. The PK parameters of area under the concentration-time curve (AUC) and maximum concentration (Cmax) were calculated by noncompartmental analysis using an internally validated software system (version 2.2.4). PK parameters and trough concentrations of PF-00547659 through 12 weeks were summarized by dose and population for comparison.

\section{Statistical Analysis}

For the overall population, statistical methods have been reported previously. ${ }^{6}$ In summary, determination of sample size was based on an assumed CDAI response rate of $35 \%$ for the placebo group and 60\% for at least one PF-00547659 dose at either week 8 or week 12 . A study population of 240 (60 in each treatment group) was estimated to provide at least a $78 \%$ probability to detect a statistically significantly greater CDAI-70 response rate with PF-00547659 compared with placebo. The CDAI remission rates in the overall population were estimated based on a generalized linear mixed model, which contained fixed factors of treatment, status of anti-TNF experience, concomitant immunosuppressive therapy, baseline CDAI value, visit, and a random effect for subject. In these subgroup analyses, data from patients enrolled at East-Asian (Japanese and Korean) study centers were pooled in the OPERA study. The analyses for the efficacy, biomarker, and PK endpoints were based on a modified intent-to-treat population, defined as all randomized subjects who received at least one dose of investigational product. The analyses for the safety endpoints were based on the safety population, which includes all enrolled subjects who received at least one dose of investigational product. For major efficacy, safety, and PK endpoints, the observed data were summarized descriptively in the Asian population. The main efficacy and safety endpoints were compared between the overall and the Asian populations.

\section{RESULTS}

\section{Disposition and Demographics}

A total of 494 subjects were screened, 265 of whom were eligible for study entry. Of the Asian subjects, 21 (8 Japanese and 13 Korean) were screened and randomized, and 20 (8 Japanese and 12 Korean) were treated as follows: 5 (0 Japanese and 5 Korean) received $22.5 \mathrm{mg}, 7$ (3 Japanese and 4 Korean) received $75 \mathrm{mg}$, and 4 (3 Japanese and 1 Korean) received 225 mg of PF-00547659, respectively, while 4 (2 Japanese and 2 Korean) received placebo. Treatment groups in the Asian population had mean ages of 22.6-33.3 years and mean CDAI total baseline scores of 298-319, whereas treatment groups in the overall population had mean ages of 34.4-37.0 years and mean CDAI total baseline scores (SD) of 307-324 (61-71). Unlike in the overall population, in which the treatment groups were similar with respect to age, weight, prior anti-TNF $\alpha$ exposure, concomitant immunosuppressant therapies, and CDAI score at baseline, no apparent similarity was observed in the Asian population except for CDAI scores due to a small number of subjects in this subpopulation (Table 1). In both overall and Asian populations, the majority of patients were treated previously with anti-TNF $\alpha$ therapies $(95.0 \%$ and $85.0 \%$ in the overall and Asian populations, respectively) and were not re- 
Table 1. Demographics and Baseline Characteristics

\begin{tabular}{|c|c|c|c|c|c|c|c|c|}
\hline \multirow{2}{*}{ Characteristic } & \multicolumn{4}{|c|}{ Overall population } & \multicolumn{4}{|c|}{ Asian population } \\
\hline & Placebo & $22.5 \mathrm{mg}$ & $75 \mathrm{mg}$ & $225 \mathrm{mg}$ & Placebo & $22.5 \mathrm{mg}$ & $75 \mathrm{mg}$ & $225 \mathrm{mg}$ \\
\hline No. & 63 & 66 & 65 & 68 & 4 & 5 & 7 & 4 \\
\hline Age (mean $\pm S D, y r)$ & $34.4 \pm 11.1$ & $37.3 \pm 13.0$ & $34.4 \pm 10.7$ & $35.9 \pm 11.0$ & $28.0 \pm 3.8$ & $22.6 \pm 2.9$ & $27.7 \pm 10.4$ & $33.3 \pm 11.7$ \\
\hline Sex, male/female & $33 / 30$ & $18 / 48$ & $30 / 35$ & $25 / 43$ & $4 / 0$ & $2 / 3$ & $6 / 1$ & $0 / 4$ \\
\hline Weight (mean $\pm S D, k g)$ & $70.1 \pm 19.4$ & $71.9 \pm 17.5$ & $69.5 \pm 21.5$ & $69.6 \pm 20.9$ & $55.1 \pm 5.2$ & $58.2 \pm 7.4$ & $57.6 \pm 8.6$ & $49.1 \pm 0.9$ \\
\hline $\begin{array}{l}\text { Relapsed after anti-TNF (secondary } \\
\text { nonresponse, \%) }\end{array}$ & 54 & 52 & 57 & 57 & 50 & 80 & 86 & 75 \\
\hline Primary anti-TNF nonresponder (\%) & 19 & 20 & 17 & 16 & 0 & 0 & 0 & 25 \\
\hline Anti-TNF intolerant (\%) & 19 & 20 & 19 & 19 & 0 & 20 & 0 & 0 \\
\hline $\begin{array}{l}\text { Failure/intolerance to } \\
\text { immunosuppressant (\%) }\end{array}$ & 8 & 9 & 8 & 7 & 50 & 0 & 14 & 0 \\
\hline Current immunosuppressant use (\%) & 33 & 41 & 43 & 38 & 25 & 20 & 57 & 75 \\
\hline Baseline CDAI (mean \pm SD) & $313 \pm 61$ & $307 \pm 71$ & $324 \pm 63$ & $316 \pm 65$ & $304 \pm 71$ & $319 \pm 50$ & $298 \pm 36$ & $317 \pm 33$ \\
\hline hs-CRP (median [01-03], mg/L) & $\begin{array}{c}18.9 \\
(8.1-30.6)\end{array}$ & $\begin{array}{c}21.1 \\
(8.0-45.8)\end{array}$ & $\begin{array}{c}14.7 \\
(6.6-32.4)\end{array}$ & $\begin{array}{c}17.2 \\
(9.5-29.2)\end{array}$ & $\begin{array}{c}37.7 \\
(18.7-70.6)\end{array}$ & $\begin{array}{c}52.3 \\
(51.0-59.3)\end{array}$ & $\begin{array}{c}26.9 \\
(14.7-50.6)\end{array}$ & $\begin{array}{c}19.9 \\
(14.2-28.8)\end{array}$ \\
\hline
\end{tabular}

hs-CRP, high-sensitivity CRP.

ceiving concomitant immunosuppressive treatment (61.1\% and 55.0\%) at randomization. Only $13(5.0 \%)$ and $3(15.0 \%)$ subjects in the overall and Asian populations, respectively, were anti-TNF $\alpha$-naïve patients; all of whom were intolerant to or had failed immunosuppressant therapy. In the overall population, the distribution of prior anti-TNF experience (failure vs. intolerance) was similar among groups, as was prior treatment with immunosuppressants. There was no clear trend regarding prior anti-TNF experience in the Asian population. However, a lower CDAI-70 response rate was observed in the $225 \mathrm{mg}$ PF-00547659 treatment group compared with the $22.5 \mathrm{mg}$ and $75 \mathrm{mg}$ groups.

\section{Primary and Secondary Endpoints}

For both the overall and the Asian populations, CDAI-70 response was not different from placebo for any treatment groups (Fig. 1A and B). However, some trends of efficacy between the overall and the Asian populations were observed. Although the number of patients in the Asian subpopulation was smaller than the overall population, a lower response rate in placebo and higher response rate in 75 mg PF-00547659 group were observed in the Asian subpopulation. A comparable trend was also observed in the secondary endpoint (CDAI-100 response) (Fig. 1C and D).

CDAI remission was observed only with $75 \mathrm{mg}$ PF-00547659 after week 4 (2/7 patients) and 22.5 mg PF-00547659 after week 8 ( $1 / 2$ patients) in the Asian population, whereas remis- sion was observed in all PF-00547659 dosing groups, including placebo, in the overall population (Fig. 2). The remission rate was not clearly dose dependent in either population.

\section{Post Hoc Analyses}

Post hoc analyses were conducted for subjects with baseline CRP $>18.8 \mathrm{mg} / \mathrm{L}$ (population median). In comparison with the results shown in Fig. 2, the remission rate at week 12 of the post hoc analyses appeared to be higher in the subjects with baseline CRP $>18.8 \mathrm{mg} / \mathrm{L}$ in the overall population (Fig. 3) considering lower remission rate with placebo in the subgroup stratified by baseline median CRP values. However, in the Asian population, no clear correlation was observed between CDAI remission rates and baseline median CRP values.

\section{Biomarker Endpoints}

In the overall population, a decline from baseline in serum hsCRP concentration was already observed for the PF-00547659 groups at week 4 (Fig. 4A). Further declines were observed in the 22.5 and $75 \mathrm{mg}$ groups at weeks 8 and 12, whereas no further decline was observed in the $225 \mathrm{mg}$ group. In the Asian population, the pattern of change of hs-CRP in the 75 and 225 mg groups was similar to that in the overall population (Fig. 4B). The level of hs-CRP fluctuated in the $22.5 \mathrm{mg}$ group.

In the overall population, circulating $\beta 7^{+}$central memory $\mathrm{CD}^{+}$T-lymphocytes increased at week 8 in a dose-dependent manner in PF-00547659-treated patients and remained ele- 
A

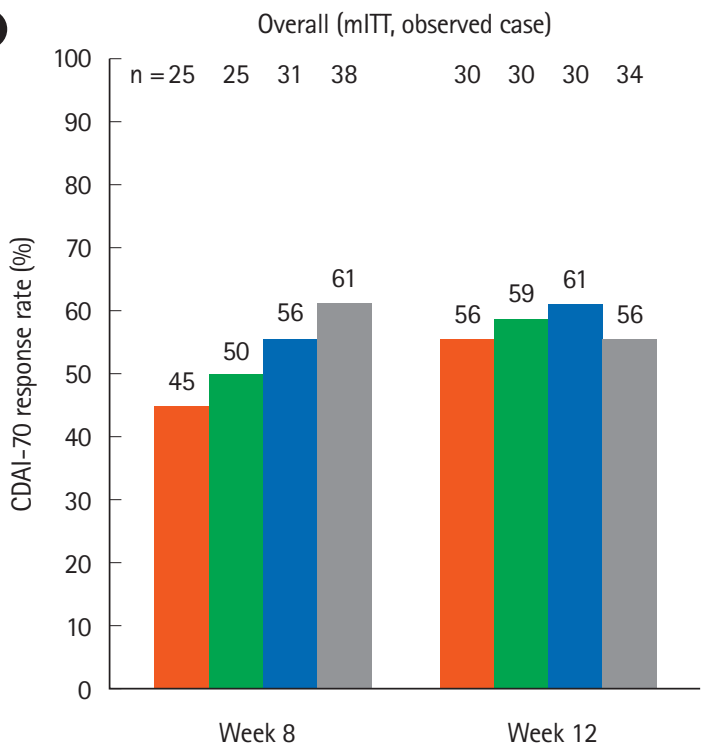

C

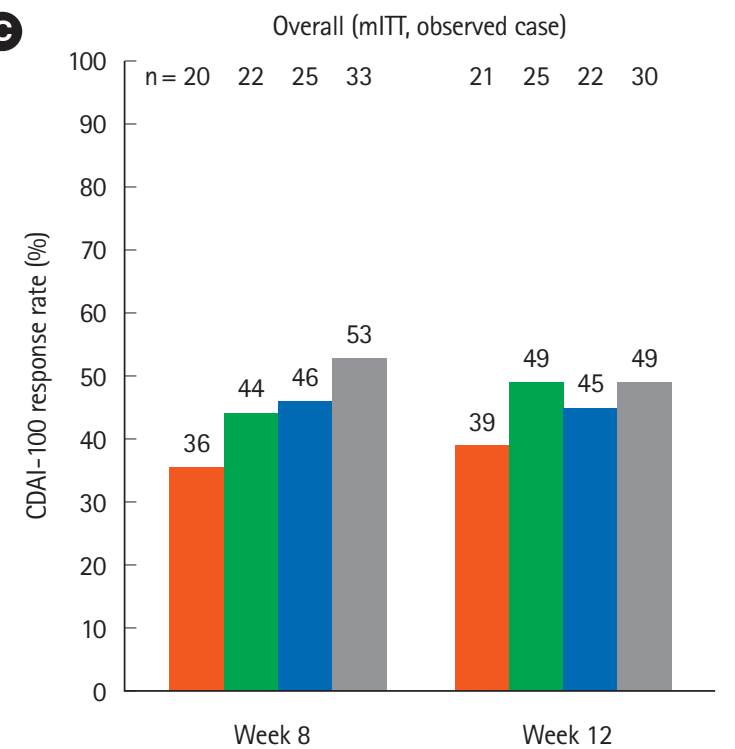

B

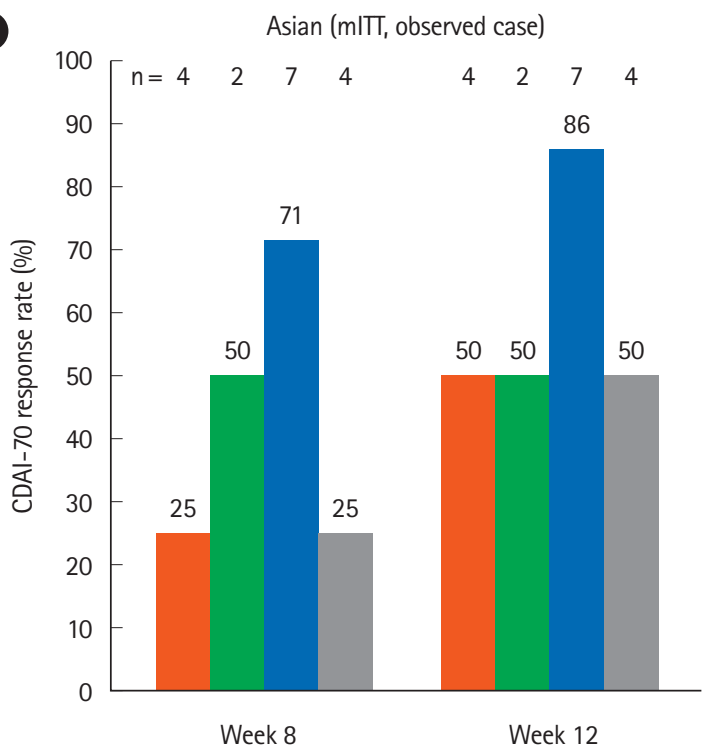

(D)

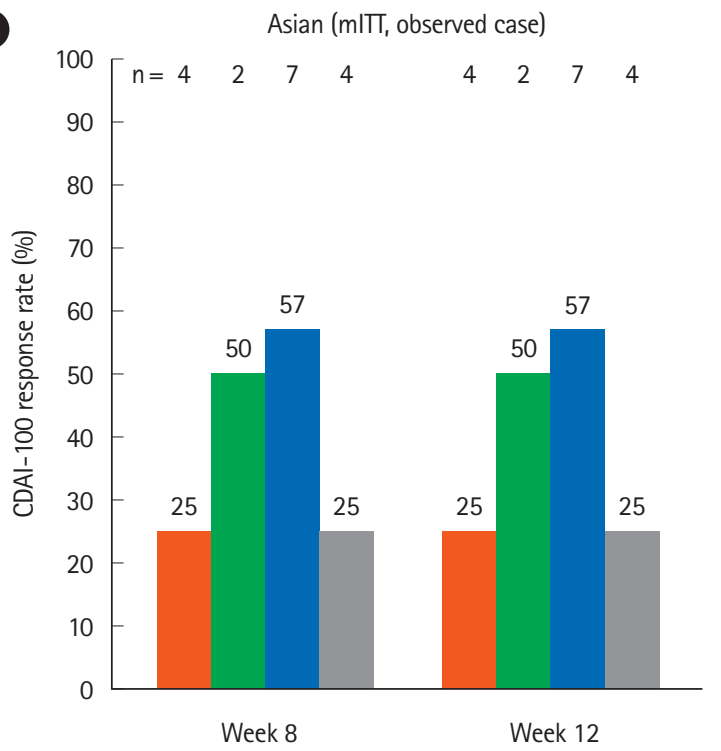

Fig. 1. Comparison of $(A, B)$ primary endpoint (CDAl-70 response rates at weeks 8 and 12$)$ and $(C, D)$ secondary endpoint (CDAl-100 response rates at weeks 8 and 12) between the $(A, C)$ overall and $(B, D)$ Asian (Japanese and Korean) populations. mIT, modified intent-to-treat population.

vated at week 12 (Fig. 4C); however, dose dependency was not maintained. In the Asian population, baseline levels of circulating $\beta 7^{+}$central memory $\mathrm{CD} 4^{+}$T-lymphocytes were about 2to 3-fold higher than those of the overall population in each treatment group (Fig. 4D). In the 75 and $225 \mathrm{mg}$ PF-00547659 groups, circulating $\beta 7^{+}$central memory $\mathrm{CD} 4^{+} \mathrm{T}$-lymphocytes in the Asian population increased in a similar fashion to the overall population at weeks 8 and 12, whereas no clear pattern was observed in $22.5 \mathrm{mg}$ PF-00547659 due to lack of data at week 12.

Soluble MAdCAM concentrations in the overall population decreased in the initial 2 weeks and the geometric mean remained $<50 \mathrm{pmol} / \mathrm{L}$ in all PF-00547659 groups, in contrast with the placebo group, in which the baseline value was maintained (Fig. 4E). Changes in soluble MAdCAM concentration in the Asian population were similar to those of the overall population (Fig. 4F). 
— Placebo 22.5 mg PF-00547659 $\quad 75$ mg PF-00547659 225 mg PF-00547659

A

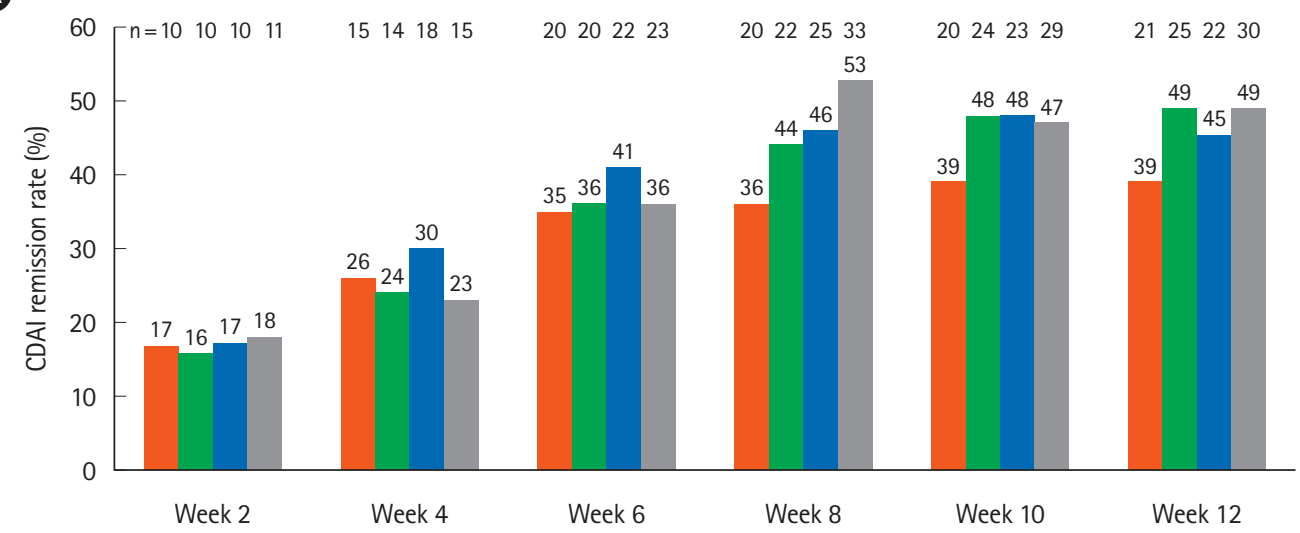

B

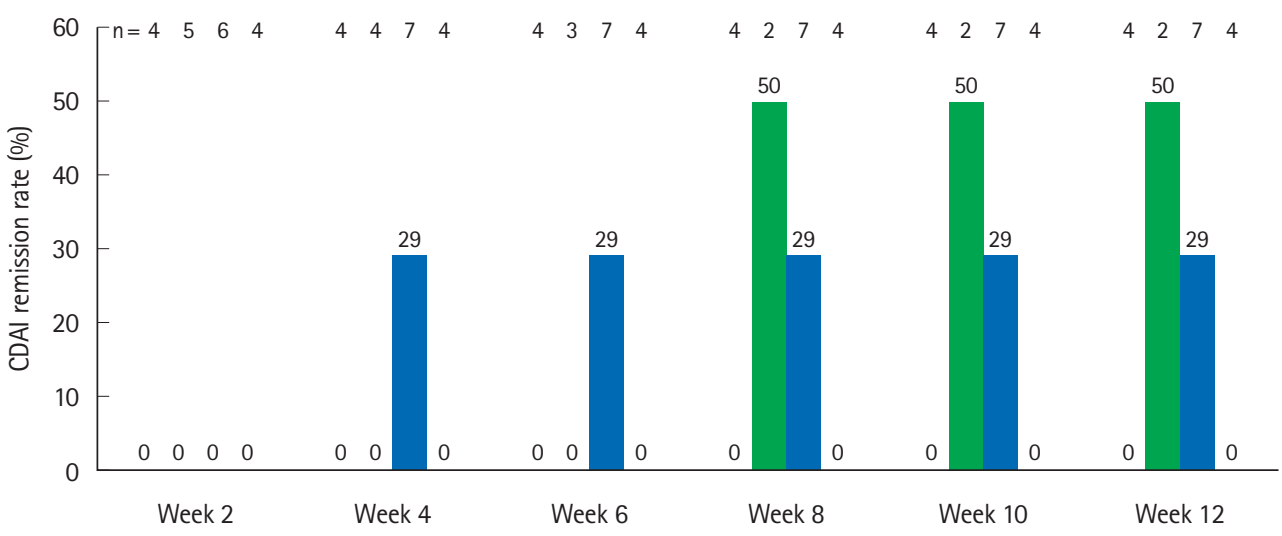

Fig. 2. Comparison of secondary endpoint (CDAl remission rates at weeks $2,4,6,8,10$, and 12) between the (A) overall and (B) Asian (Japanese and Korean) populations. mIT, modified intent-to-treat population.
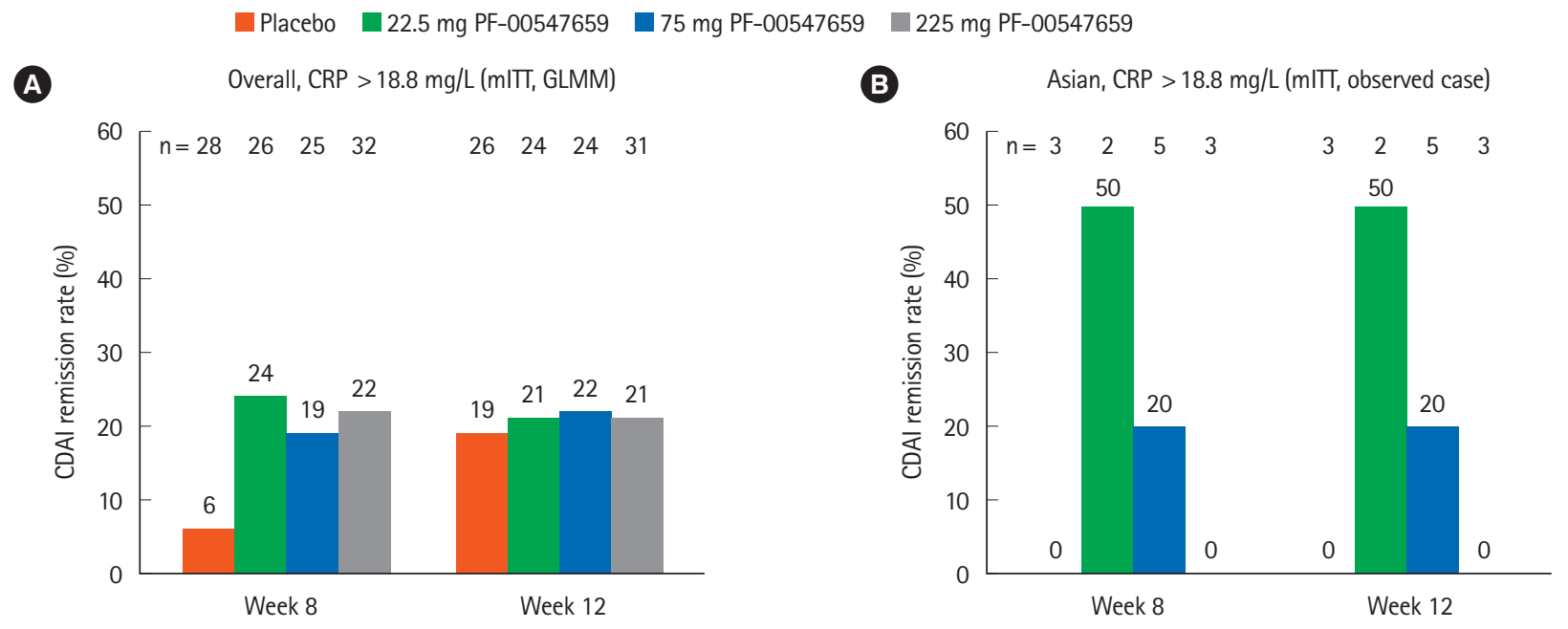

Fig. 3. Results of the post hoc analysis (CDAl remission rates at weeks 8 and 12) between the (A) overall and (B) Asian (Japanese and Korean) populations with baseline CRP > $18.8 \mathrm{mg} / \mathrm{L}$. The generalized linear mixed model (GLMM) contains fixed factors of treatment, the status of antiTNF experience, the concomitant immunosuppressive therapy, baseline value, visit and treatment by visit, and a random effect of subject. mIT, modified intent-to-treat population. 


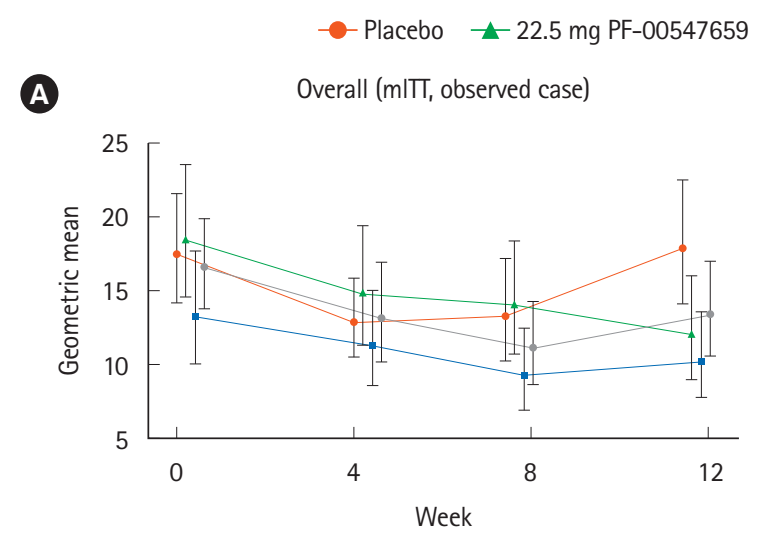

75 mg PF-00547659 - -225 mg PF-00547659
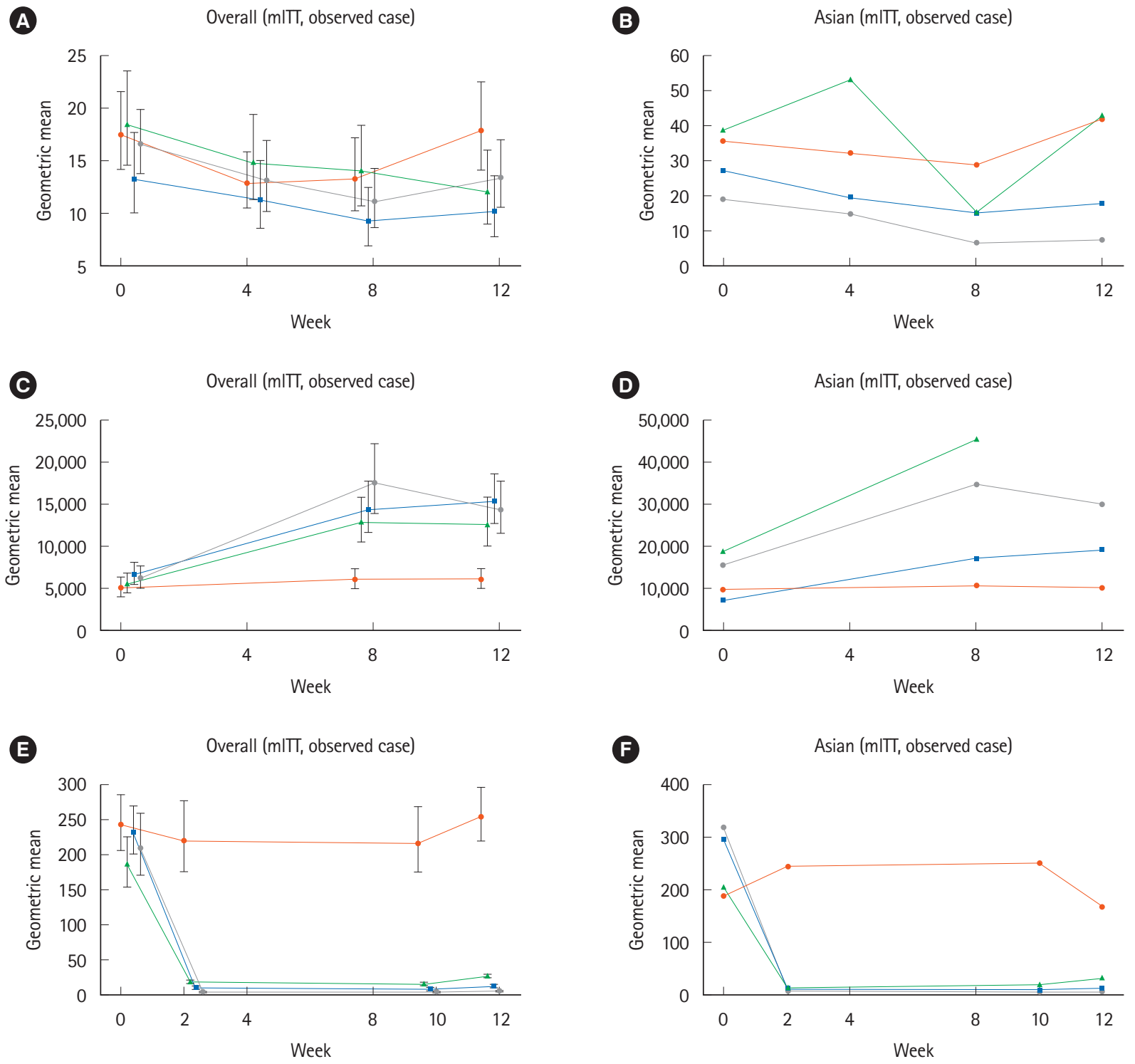

Fig. 4. Changes of hs-CRP at weeks $0,4,8$, and $12(A, B)$, percentage of $\beta 7^{+}$central memory $C D 4^{+}$T-lymphocytes ${ }^{*} M E S F$ at weeks 0,8 , and $12(C$, $D)$, and changes of soluble MAdCAM at weeks $0,2,10$, and $12(E, F)$ in the $(A, C, E)$ overall and $(B, D, F)$ Asian (Japanese and Korean) populations. Error bars in the overall population denote 90\% Cls. hs-CRP, high-sensitivity CRP; MESF, Molecules of Equivalent Soluble Fluorochrome; MAdCAM, mucosal addressin cell adhesion molecule; mIT, modified intent-to-treat population.

\section{PK and ADAs}

For PK exposures (AUC648 and Cmax) in non-Japanese subjects, there was a greater than dose-proportional increase between 22.5 to $75 \mathrm{mg}$ but a slightly less than dose-proportional increase from 75 to $225 \mathrm{mg}$, likely due to saturation of targetmediated clearance at higher doses (Table 2). This trend was not seen in the PK analyses of Japanese subjects at doses of 75 and $225 \mathrm{mg}$, which may be due to the much higher exposure observed at $225 \mathrm{mg}$ in Japanese subjects compared with non-
Japanese subjects. Indeed, in the limited number of Japanese subjects, the ratios of AUC648 and Cmax of Japanese/nonAsian were 0.74 and 0.83 , respectively, at $75 \mathrm{mg}$; and 1.63 and 1.65, respectively, at $225 \mathrm{mg}$. Median time to maximum plasma concentration (Tmax) ranged from 140 to 165 hours, with no difference between Japanese and non-Japanese subjects. Half-life $\left(\mathrm{t}^{1 / 2}\right)$ tended to be longer at higher doses than the lower dose of $22.5 \mathrm{mg}$. No apparent difference was seen for $\mathrm{t}^{1 / 2} \mathrm{be}$ tween the 2 populations. 
Table 2. Descriptive Summary of Serum PK Parameters of PF-00547659 in Japanese and Non-Japanese CD Subjects

\begin{tabular}{|c|c|c|c|c|c|c|c|}
\hline \multirow{3}{*}{ PK parameter } & \multicolumn{7}{|c|}{ Treatment } \\
\hline & \multirow{2}{*}{$\begin{array}{c}22.5 \mathrm{mg} \\
\begin{array}{c}\text { Non-Japanese } \\
(n=7)\end{array}\end{array}$} & \multicolumn{3}{|c|}{$75 \mathrm{mg}$} & \multicolumn{3}{|c|}{$225 \mathrm{mg}$} \\
\hline & & $\begin{array}{c}\text { Japanese } \\
(\mathrm{n}=3)\end{array}$ & $\begin{array}{c}\text { Non-Japanese } \\
(n=7)\end{array}$ & Ratio $^{a}$ & $\begin{array}{c}\text { Japanese } \\
(n=3)\end{array}$ & $\begin{array}{c}\text { Non-Japanese } \\
(n=10)\end{array}$ & Ratio $^{a}$ \\
\hline $\mathrm{AUC}_{648}(\mu \mathrm{g} \cdot \mathrm{hr} / \mathrm{mL})$ & 550 (53) & 3,399 (16) & 4,620 (32) & 0.74 & $15,630(39)$ & $9,608(41)^{b}$ & 1.63 \\
\hline $\mathrm{C} \max (\mu \mathrm{g} / \mathrm{mL})$ & $1.756(45)$ & $9.454(26)$ & $11.430(19)$ & 0.83 & $35.360(15)$ & $21.480(47)$ & 1.65 \\
\hline Tmax (hr) & $140(44-333)$ & $144(125-170)$ & $142(45-171)$ & - & $165(144-166)$ & $154(52-214)$ & - \\
\hline $\mathrm{t} 1 / 2$ (day) & $4.977 \pm 2.844^{c}$ & $7.907 \pm 1.713$ & $9.288 \pm 2.713^{d}$ & - & $7.870 \pm 11.900^{e}$ & $13.380 \pm 4.040^{f}$ & - \\
\hline
\end{tabular}

Geometric mean (geometric \%CV) for all except: median (range) for Tmax; arithmetic mean \pm SD for $\mathrm{t} 1 \frac{2}{2}$.

${ }^{a}$ Ratio = Japanese/non-Japanese (no pharmacokinetics [PK] parameter available from other Asian subjects).

b $(n=9)$.

' $(n=6)$.

d $(n=5)$.

endividual subject values are presented for $<3$ subjects.

$\mathrm{f}(\mathrm{n}=4)$.

AUC, area under the concentration-time curve; Cmax, maximum concentration; Tmax, median time to maximum plasma concentration, $\mathrm{t} 1 / 2$, half-life; $C V$, coefficient of variation.

Table 3. Number of AEs and Relevant Discontinuations Including Detailed Numbers of Infections in the Overall and Asian (Japanese and Korean) Populations

\begin{tabular}{|c|c|c|c|c|c|c|c|c|c|c|}
\hline \multirow[t]{2}{*}{ Population } & \multicolumn{2}{|c|}{ Placebo } & \multicolumn{2}{|c|}{$22.5 \mathrm{mg}$} & \multicolumn{2}{|c|}{$75 \mathrm{mg}$} & \multicolumn{2}{|c|}{$225 \mathrm{mg}$} & \multicolumn{2}{|c|}{$\begin{array}{l}\text { All active } \\
\text { treatment }\end{array}$} \\
\hline & Overall & Asian & Overall & Asian & Overall & Asian & Overall & Asian & Overall & Asian \\
\hline No. & 63 & 4 & 66 & 5 & 65 & 7 & 68 & 4 & 199 & 16 \\
\hline Subjects with AEs & $54(86)$ & $3(75)$ & $57(85)$ & $4(80)$ & $51(80)$ & $4(57)$ & $53(78)$ & $4(100)$ & $161(81)$ & $12(75)$ \\
\hline Subjects with serious AEs & $5(8)$ & 0 & $11(16)$ & $1(20)$ & $9(14)$ & 0 & $11(16)$ & 0 & $31(16)$ & $1(6)$ \\
\hline Discontinuations because of $\mathrm{AE}$ & $3(5)$ & 0 & $9(13)$ & $2(40)$ & $8(13)$ & 0 & $4(6)$ & 0 & $21(11)$ & $2(13)$ \\
\hline$C D$ & $3(5)$ & 0 & $4(6)$ & 0 & $3(5)$ & 0 & $2(3)$ & 0 & $9(5)$ & 0 \\
\hline CD-related & 0 & 0 & $2(3)$ & $1(20)$ & $3(5)$ & 0 & $1(1)$ & 0 & 6 (3) & $1(6)$ \\
\hline Other & 0 & 0 & $3(4)$ & $1(20)$ & $2(3)$ & 0 & $1(1)$ & 0 & $6(3)$ & $1(6)$ \\
\hline Infections & $20(32)$ & $1(25)$ & $27(40)$ & $1(20)$ & $25(39)$ & $2(29)$ & $24(35)$ & $3(75)$ & $76(38)$ & $6(38)$ \\
\hline $\mathrm{Gl}$ tract/liver infections ${ }^{\mathrm{a}}$ & $1(2)$ & 0 & $8(12)$ & 0 & $7(11)$ & $1(14)$ & $4(6)$ & 0 & $19(10)$ & $1(6)$ \\
\hline $\begin{array}{l}\text { Infections in ex-GI MAdCAM bearing } \\
\text { tissues }\end{array}$ & $10(16)$ & 0 & $6(9)$ & 0 & $6(9)$ & $1(14)$ & $8(12)$ & $2(50)$ & $20(10)$ & $3(19)$ \\
\hline Nasopharyngitis & $5(8)$ & 0 & $3(4)$ & 0 & $4(6)$ & $1(14)$ & $5(7)$ & $1(25)$ & $12(6)$ & $2(13)$ \\
\hline Bladder & $5(8)$ & 0 & $3(4)$ & 0 & $2(3)$ & 0 & $3(4)$ & $1(25)$ & $8(4)$ & $1(6)$ \\
\hline Other (spleen, uterus) & 0 & 0 & 0 & 0 & 0 & 0 & 0 & 0 & 0 & 0 \\
\hline
\end{tabular}

Values are presented as number (\%).

${ }^{a}$ Anal abscess, anal fistula infection, appendicitis. Gastroenteritis, gastroenteritis viral, Gl inflammation, GI infection, GI viral infection, liver abscess, perirectal abscess, and rectal abscess (MedDRA version 18.1). ${ }^{10}$

$A E$, adverse event; MAdCAM, mucosal addressin cell adhesion molecule.

Mean trough concentrations of PF-00547659 at week 12 were generally similar between Asian (Japanese and Korean subjects) and overall subjects ( $3.49 \mu \mathrm{g} / \mathrm{mL}$ vs. $4.87 \mu \mathrm{g} / \mathrm{mL}$ at $75 \mathrm{mg}$ and $20.22 \mu \mathrm{g} / \mathrm{mL}$ vs. $17.30 \mu \mathrm{g} / \mathrm{mL}$ at $225 \mathrm{mg}$ dose, for Asians vs. overall subjects, respectively). PF-00547659 con- centration-time plots indicated no effect of positive ADAs on exposure (data not shown).

\section{Safety}

In both the overall and the Asian populations, there was no 
difference in the frequency of AEs between placebo and PF00547659 treatment groups and no evidence for a dose-related increase in AEs (Table 3).$^{10}$ In the overall population, discontinuations due to AEs were higher in the 22.5 and $75 \mathrm{mg}$ PF-00547659 groups (9 and 8, respectively) than in the placebo and $225 \mathrm{mg}$ PF-00547659 groups (3 and 4, respectively). Most AE withdrawals were due to $\mathrm{CD}$ or its complications. There was no evidence of a PF-00547659 drug or dose relationship. In addition, serious adverse events (SAEs) were reported more frequently in patients receiving PF-00547659 than those given placebo, with no evidence of a dose response. Reported SAEs appeared unlikely to be due to the study drug. Overall rates of infections and non-GI infections were comparable between the placebo and the combined PF-00547659 treatment groups; however, higher rates were observed for GI infections in the PF-00547659 treatment groups versus the placebo group, although there was no evidence of dose relationship. Overall, the incidence of AEs, SAEs, and discontinuations were similar across treatment groups, with no evidence of an increased AE rate at higher doses of PF-00547659.

\section{DISCUSSION}

As is the case with the overall population, the differences in the rates of CDAI- 70 response, $\mathrm{CDAI}-100$ response, and CDAI remission between PF-00547659 and placebo did not show a difference at weeks 8 or 12 in the Asian population in this study. However, for CDAI-70 and CDAI-100, there were numerically lower response rates with placebo in the Asian population than in the overall population. There was a lower CDAI-70 response rate in the $225 \mathrm{mg}$ PF-00547659 group in the Asian population compared with the $22.5 \mathrm{mg}$ and $75 \mathrm{mg}$ groups. The explanation for the lower response rate may be due to the high non-responder rate (total 100\% after primary and secondary treatments) to anti-TNF experience and high proportion (75\%) of immunosuppressant use in the $225 \mathrm{mg}$ group, which may result in a lower overall hs-CRP at baseline compared to other treatment groups. Considering the precedent that objective evidence of inflammation at baseline in the overall population brings lower placebo response and remission rates, ${ }^{11-15}$ the 2-fold higher hs-CRP value observed in the placebo group of the Asian population (37.7) compared with the overall population (18.9) may have contributed to the lower rates of CDAI-70 and CDAI-100 responses in placebo. This is also supported by the results of the post hoc analysis in this study that the lower response rates in placebo are observed in the subjects in the overall population with baseline CRP $>18.8 \mathrm{mg} / \mathrm{L}$ (Fig. 4). On the other hand, the higher response rate of CDAI-70 observed in the $75 \mathrm{mg}$ PF-00547659 group in the Asian population compared with the overall population (Fig. 1) has no clear explanation. Furthermore, while patients with higher levels of inflammation as measured by baseline hsCRP, or more active disease as measured by SES-CD, may have a better response to treatment with PF-00547659 in the overall population, ${ }^{5}$ no such findings were observed in the Asian population. In order to better understand these findings, more data and analyses of efficacy of PF-00547659 are required in Asian (Japanese and Korean) CD patients.

In contrast to the lack of direct evidence of clinical efficacy based on the primary and secondary endpoints, decline in serum hs-CRP concentration, increase in circulating $\beta 7^{+}$central memory $\mathrm{CD}^{+}$T-lymphocytes, and a change in soluble MAdCAM were evidently observed in the PF-00547659 groups in both the overall and the Asian (Japanese and Korean) populations, demonstrating that PF-00547659 was pharmacologically active in both populations. There is no clear explanation for the observation of higher values of circulating $\beta 7^{+}$central memory $\mathrm{CD}^{+}$T-lymphocytes at baseline in the Asian population, but it might be one of ethnic differences in CD.

In the analysis of PK, PF-00547659 showed nonlinear PK in CD patients after subcutaneous dosing, which is consistent with that displayed by IgG monoclonal antibody with targetmediated disposition. ${ }^{16}$ Japanese subjects showed slightly lower exposure (AUC648 and Cmax) at the $75 \mathrm{mg}$ dose, but much higher exposure at the $225 \mathrm{mg}$ dose, compared with non-Japanese subjects. This may simply reflect inter-subject variability in the small population studied. However, the much lower body weight for Asian subjects at $225 \mathrm{mg}$ dose (mean value of $49.1 \mathrm{~kg}$, including 3 Japanese out of 4 Asian subjects) might be another reason, and body weight has generally been included in the population PK analysis as a covariate, especially for biologic compounds. ${ }^{17}$ Trough levels of PF-00547659 were generally comparable between Asian and overall subjects, considering the relatively small subject number and large PK variability.

No safety signal was observed in the overall or the Asian populations. Under the very limited numbers of subjects, all the PF-00547659 doses appeared to be safe, and no safety signals were observed in any of the groups in the Asian population up to 12 weeks of treatment by PF-00547659. Taken together with the results of the overall population, the safety profile of PF-00547659 appears acceptable in both the overall and the Asian populations for up to 12 weeks of treatment. 
However, a longer evaluation period exceeding 12 weeks is required in order to understand more fully the safety profile of PF-00547659 in treatment of CD patients.

No clear conclusion can be drawn for any potential correlation between level of inflammation and clinical efficacy in the Asian population, unlike the overall population. PF-00547659 biological activity was observed in the overall and the Asian populations based on changes in serum hs-CRP concentration, increase in circulating $\beta 7^{+}$central memory T-lymphocytes, and change in soluble MAdCAM. PK of PF-00547659 was generally comparable between Asian and non-Asian subjects. The safety profile of PF-00547659 appears acceptable in both populations up to 12 weeks of treatment. The main limitation of this study was the small number of subjects in the Asian population, which did not allow clear conclusions to be drawn. A longer treatment period exceeding 12 weeks with adequate numbers of subjects is required for better understanding of efficacy, safety, and PK profiles of PF-00547659 in Asian CD patients.

\section{FINANCIAL SUPPORT}

This study was sponsored by Pfizer. Medical writing support was provided by Sharmy Blows of Engage Scientific, Horsham, UK, and was funded by Pfizer.

\section{CONFLICT OF INTEREST}

This study was sponsored by Pfizer. Banerjee A, Isogawa N, Li Y, and Hassan-Zahraee M are employees of Pfizer. Ahmad A, Cataldi F, Gorelick KJ, Nagaoka M, and Clare R were employees of Pfizer during the OPERA study. Saruta M received lecture fees from Mitsubishi Tanabe Pharma, AbbVie GK, and Takeda Pharmaceutical Co., Ltd., and research grants from EA Pharma Co., Ltd., Kissei Pharmaceutical Co., Ltd., Otsuka Pharmaceutical Co., Ltd., and Mochida Pharmaceutical Co., Ltd. Watanabe $\mathrm{M}$ has the following disclosures: honoraria from Mitsubishi Tanabe Pharma Corp., Eisai Co., Ltd., Kyorin Pharmaceutical Co., Ltd., JIMRO Co., Ltd., AbbVie GK, Takeda Pharmaceutical Co., Ltd., Kyowa Hakko Kirin Co., Ltd., Zeria Pharmaceutical Co., Ltd., Asahi Kasei Medical Co., Ltd., EA Pharma Co., Ltd., Astellas Pharma Inc., Mochida Pharmaceutical Co., Ltd., Janssen Pharmaceutical Co., Ltd., Gilead Sciences, Inc., Celgene Corp., KISSEI Pharmaceutical Co., Ltd., and commercial research funding from: Asahi Kasei Medical Co., Ltd., AbbVie GK, EA Pharma Co., Ltd., Eisai Co., Ltd., Kyorin Phar- maceutical Co., Ltd., Mitsubishi Tanabe Pharma Corp., Otsuka Pharmaceutical Co., Ltd., Kyowa Hakko Kirin Co., Ltd., Zeria Pharmaceutical Co., Ltd., JIMRO Co., Ltd., Takeda Pharmaceutical Co., Ltd., Nippon Kayaku Co., Ltd., Mochida Pharmaceutical Co., Ltd., Daiichi Sankyo Co., Ltd., Astellas Pharma Inc., MSD K.K., Dainippon Sumitomo Dainippon Pharma Co., Ltd., Bristol-Myers, K.K, Chugai Pharmaceutical Co., Ltd., Gilead Sciences, Inc., Pfizer Inc., Miyarisan Pharmaceutical Co., Ltd., KISSEI Pharmaceutical Co., Ltd., Taiho Pharmaceutical Co., Ltd. Jang BI, Park DI, Cheon JH, Im JP, Yang SK, Hibi T, Kanai T, Katsuno T, Kim YH, and Li Y have no disclosures. However, all of these are not relevant to this article.

\section{AUTHOR CONTRIBUTION}

All authors contributed to the design of the study and the acquisition, analysis, or interpretation of the data. All authors contributed to the development of the manuscript and provided approval of the final version for submission.

\section{ORCID}

$\begin{array}{ll}\text { Saruta M } & \text { https://orcid.org/0000-0001-8172-3240 } \\ \text { Park DI } & \text { https://orcid.org/0000-0003-2307-8575 } \\ \text { Kim YH } & \text { https://orcid.org/0000-0003-1803-2513 } \\ \text { Yang SK } & \text { https://orcid.org/0000-0003-2772-2575 } \\ \text { Jang BI } & \text { https://orcid.org/0000-0002-3037-9272 } \\ \text { Cheon JH } & \text { https://orcid.org/0000-0002-2282-8904 } \\ \text { Im JP } & \text { https://orcid.org/0000-0003-1584-0160 } \\ \text { Kanai T } & \text { https://orcid.org/0000-0002-1466-4532 } \\ \text { Katsuno T } & \text { https://orcid.org/0000-0003-1346-0893 } \\ \text { Ishiguro Y } & \text { https://orcid.org/0000-0002-0429-5956 } \\ \text { Nagaoka M } & \text { https://orcid.org/0000-0001-7193-1548 } \\ \text { Isogawa N } & \text { https://orcid.org/0000-0003-4529-4834 } \\ \text { Li Y } & \text { https://orcid.org/0000-0002-6161-0000 } \\ \text { Ahmad A } & \text { https://orcid.org/0000-0002-6935-6748 } \\ \text { Hassan-Zahraee M } & \text { https://orcid.org/0000-0003-1951-8304 } \\ \text { Clare R } & \text { https://orcid.org/0000-0001-9689-7550 } \\ \text { Gorelick KJ } & \text { https://orcid.org/0000-0002-0481-5739 } \\ \text { Cataldi F } & \text { https://orcid.org/0000-0002-8738-8989 } \\ \text { Watanabe M } & \text { https://orcid.org/0000-0002-5475-9544 } \\ \text { Hibi T } & \text { https://orcid.org/0000-0002-6256-1204 }\end{array}$

\section{ACKNOWLEDGEMENTS}

The authors wish to thank Dr Reiko Kunisaki and Dr Tomohi- 
ro Kudo for their contributions to this manuscript. The authors also wish to thank the patients who participated in the study, and Dr Geun Am Song, the investigators and medical staff of all participating study centers. They would also like to acknowledge Steven Martin for comments on an earlier version of this paper, and Dr Gail M Comer for her contributions to the conception and design of the study as well as the members of the Data Monitoring Committee: Dr Colm O'Morain (Chair), Dr Paola Cinque, Dr David Clifford, Dr Lauren Krupp, Dr Glen Cooke, and Dr Dan Anbar.

\section{REFERENCES}

1. Fiocchi C. Inflammatory bowel disease pathogenesis: where are we? J Gastroenterol Hepatol 2015;30 Suppl 1:12-18.

2. Streeter PR, Berg EL, Rouse BT, Bargatze RF, Butcher EC. A tissue-specific endothelial cell molecule involved in lymphocyte homing. Nature 1988;331:41-46.

3. Nakache M, Berg EL, Streeter PR, Butcher EC. The mucosal vascular addressin is a tissue-specific endothelial cell adhesion molecule for circulating lymphocytes. Nature 1989;337:179181.

4. Briskin M, Winsor-Hines D, Shyjan A, et al. Human mucosal addressin cell adhesion molecule-1 is preferentially expressed in intestinal tract and associated lymphoid tissue. Am J Pathol 1997;151:97-110.

5. Pullen N, Molloy E, Carter D, et al. Pharmacological characterization of PF-00547659, an anti-human MAdCAM monoclonal antibody. Br J Pharmacol 2009;157:281-293.

6. Sandborn WJ, Lee SD, Tarabar D, et al. Phase II evaluation of anti-MAdCAM antibody PF-00547659 in the treatment of Crohn's disease: report of the OPERA study. Gut 2018;67:1824-
1835.

7. Ng SC. Epidemiology of inflammatory bowel disease: focus on Asia. Best Pract Res Clin Gastroenterol 2014;28:363-372.

8. Sands BE, Katz S, Wolf DC, et al. A randomised, double-blind, sham-controlled study of granulocyte/monocyte apheresis for moderate to severe Crohn's disease. Gut 2013;62:1288-1294.

9. Park SC, Jeen YT. Genetic studies of inflammatory bowel disease-focusing on Asian patients. Cells 2019;8:E404.

10. Introductory guide MedDRA version 18.1, 2015. MedDRA Web site. https://www.meddra.org/sites/default/files/guidance/file/intguide_18_1_english.pdf. Accessed Jan 16, 2020.

11. Sandborn WJ, Feagan BG, Rutgeerts P, et al. Vedolizumab as induction and maintenance therapy for Crohn's disease. N Engl J Med 2013;369:711-721.

12. Schreiber S, Rutgeerts $P$, Fedorak RN, et al. A randomized, placebo-controlled trial of certolizumab pegol (CDP870) for treatment of Crohn's disease. Gastroenterology 2005;129:807818.

13. Targan SR, Feagan BG, Fedorak RN, et al. Natalizumab for the treatment of active Crohn's disease: results of the ENCORE Trial. Gastroenterology 2007;132:1672-1683.

14. Sandborn WJ, Schreiber S, Feagan BG, et al. Certolizumab pegol for active Crohn's disease: a placebo-controlled, randomized trial. Clin Gastroenterol Hepatol 2011;9:670-678.

15. Colombel JF, Sandborn WJ, Reinisch W, et al. Infliximab, azathioprine, or combination therapy for Crohn's disease. N Engl J Med 2010;362:1383-1395.

16. Mager DE. Target-mediated drug disposition and dynamics. Biochem Pharmacol 2006;72:1-10.

17. Wang DD, Zhang S, Zhao H, Men AY, Parivar K. Fixed dosing versus body size-based dosing of monoclonal antibodies in adult clinical trials. J Clin Pharmacol 2009;49:1012-1024. 